\title{
Schiffbau in Europa - Europäische Kooperation als Antwort auf die Globalisierung?
}

\author{
Thorsten Ludwig \\ Jochen Tholen
}

\begin{abstract}
Dem europäischen Schiffbau geht es so gut wie lange nicht mehr, denn die Auftragsbücher versprechen Arbeit bis über das Jahr 2010 hinaus. Gleichwohl ist die Wettbewerbsfähigkeit der Schiffbauindustrie bedroht durch zwei strukturelle Defizite: Unterentwickelte horizontale Kooperation und akuter Fachkräftemangel auf allen Qualifikationsniveaus. Angesichts der Konkurrenz aus Südostasien muss der Schiffbau als Kern der maritimen Industrie Europas diesen Herausforderungen begegnen, wenn er auch nach 2010 seine Wettbewerbsfähigkeit erhalten will. Hierfür müssten eine bessere Zusammenarbeit zwischen den Werften und eine strategisch orientierte Personal- und Qualifizierungspolitik realisiert werden.
\end{abstract}

\section{Problemaufriss}

Die europäische Schiffbauindustrie erlebt seit dem Jahr 2003 einen Auftragsboom, der die Beschäftigung bis über das Jahr 2010 hinaus sichert. Nach einem krisenhaften Jahrzehnt mit Beschäftigungsabbau und Werftensterben verfügen die Werften damit über ein komfortables Auftragspolster. Dieses eröffnet ihnen die Möglichkeit, sich auf den prognostizierten Auftragsrückgang im Weltschiffbau ab dem Jahr 2008 vorzubereiten und die Strukturen des europäischen Schiffbaus mit dem Ziel der Stärkung der Wettbewerbsfähigkeit zu verbessern.

Der Erhalt einer europäischen Schiffbauindustrie als maritime Schlüsselindustrie und High-Tech-Branche ist nicht nur für den maritimen Sektor, sondern für die europäische Wirtschaft insgesamt von hoher Bedeutung. Allein 3 bis $5 \%$ des europäischen Bruttoinlandsprodukts werden durch meeresspezifische Industrien und Dienstleistungen erwirtschaftet. Beinahe $90 \%$ aller Außenhandelsgüter und über $40 \%$ der Binnenhandelsgüter der EU werden auf dem Seeweg transportiert. Darüber hinaus werden in den 1.200 europäischen Häfen jedes Jahr 3,5 Mrd. Tonnen Fracht umgeschlagen und über 350 Mio. Passagiere abgefertigt, und mit einem 40\%igen Anteil an der Welthandelsflotte nimmt Europa die Spitzenposition ein (Kommission der Europäischen Gemeinschaften 2006, S. 7).

Der europäische Schiffbau muss drei zentralen Herausforderungen begegnen: Erstens werden vor allem in Südostasien neue Produktionskapazitäten aufgebaut, die in absehbarer Zeit zu deutlichen Überkapazitäten im Weltschiffbau führen werden. China wird zukünftig den Wettbewerb im Weltschiffbau nachhaltig beeinflussen, denn gemäß den Plänen der chinesischen Regierung soll das Land spätestens im Jahr 2015 die größte Schiffbaunation der Welt sein. Mitte des 21. Jahrhunderts - so die Vorgaben - will China die Hälfte der weltweiten Nachfrage nach Schiffen decken (EMF 2006, S. 31-43). Zweitens wird besonders in China und Südkorea der Schiffbau in vielfältiger Weise subventioniert, sodass es auch dadurch zu Wettbewerbsverzerrungen kommt. Drittens drängen die dominierenden Schiffbaunationen Südkorea, Japan und China in Schiffssegmente vor, in denen bislang die europäischen Werften führend sind. ${ }^{1}$

Die europäische Schiffbauindustrie positioniert sich im internationalen Wettbewerb durch innovative Schiffskonzepte und Produktionsprozesse, welche ihr gegenüber den südostasiatischen Konkurrenten insbesondere in speziellen Marktsegmenten einen Vorsprung verschaffen. Offen ist jedoch die Frage, inwieweit die Kooperation zwischen den europäischen Werften sowie eine proaktive Personalund Qualifizierungspolitik einen Beitrag zur aktuell relativ guten Marktstellung des europäischen Schiffbaus zu leisten vermochten und ob eine Intensivierung von Zusammenarbeit und Personalplanung die Werften im internationalen Wettbewerb weiter stärken würde. In diesem Kontext ist ebenfalls zu prüfen, ob eine europäische Industriepolitik Kooperation und Qualifizierung in geeigneter Form unterstützen kann.

\footnotetext{
Vor diesem Hintergrund führte das Institut Arbeit und Wirtschaft (IAW) der Universität und der Arbeitnehmerkammer Bremen ein Forschungsprojekt von November 2003 bis Dezember 2006 durch, welches die Struktur des europäischen Schiffbaus und dessen Zukunftsperspektiven untersuchte. Das Forschungsprojekt "Schiffbau in Europa“ wurde finanziert durch die Hans-BöcklerStiftung (HBS), die Otto-Brenner-Stiftung (OBS) und das Rationalisierungs- und Innovationszentrum der deutschen Wirtschaft (RKW). Methodisch basiert das Projekt auf einer Befragung aller 240 europäischen Werften in 20 europäischen Schiffbauländern, drei Fallstudien, die sich auf den Containerschiffbau, den Kreuzfahrtschiffbau und den Marineschiffbau in Europa konzentrierten, und über 60 Interviews mit Vertretern von 22 der wichtigsten Werften Europas. Das Projekt wurde gleichzeitig vom Europäischen Metallgewerkschaftsbund (EMB) und dem Committee of European Shipyard's Associations (CESA) unterstützt. Es war zudem Bestandteil des Sozialen Dialogs Schiffbau auf EU-Ebene, der ausgewählte Ergebnisse der quantitativen Befragung im März 2006 in einer Sonderpublikation veröffentlicht hat (siehe auch Tholen/Ludwig 2006).
}

Thorsten Ludwig, Dipl.-Pol., wissenschaftlicher Mitarbeiter am Institut Arbeit und Wirtschaft (IAW) der Universität Bremen. Arbeitsschwerpunkte: Maritime Wirtschaft, Industrielle Beziehungen, Wandel der Arbeitsgesellschaft. e-mail: ludwig@iaw.uni-bremen.de Jochen Tholen, Dr., Leiter des Forschungsschwerpunktes "Wirtschaft und Finanzen" des Instituts Arbeit und Wirtschaft (IAW) der Universität Bremen. Arbeitsschwerpunkte: Maritime Wirtschaft, European Labour Relations, Human Ressource Management, Eliten, Strukturpolitik.

e-mail: tholen@iaw.uni-bremen.de 


\section{Entwicklung des europäischen Schiffbaus}

\subsection{PRODUKTION UND BESCHÄFTIGUNG}

In den 1960er Jahren war Westeuropa mit einem Marktanteil von bis zu $40 \%$ am Weltschiffbau noch die mit Abstand wichtigste Schiffbauregion der Welt. Die Ölkrise der 1970er Jahre führte jedoch zu einem signifikanten Einbruch im Schiffbau, von dem nicht nur Europa, sondern sämtliche Schiffsproduzenten weltweit betroffen waren. In den darauf folgenden Jahrzehnten erholte sich der Weltschiffbaumarkt zusehends, allerdings gelang es Europa nicht, seinen Marktanteil wesentlich zu steigern. Entgegengesetzt verlief die Entwicklung in den heute führenden Schiffbaunationen Japan und Südkorea: Sie stellten den Schiffbau in das Zentrum ihrer entwicklungsund industriepolitischen Strategien und förderten diese Industrie mit beachtlichen finanziellen und politischen Initiativen. So führte im Jahr 2005 Südkorea die Rangliste der wichtigsten Schiffbaunationen mit einem Weltmarktanteil von $37,7 \%$ an. Auf Rang zwei folgte Japan mit $35 \%$, China lag mit 13,9\% auf Platz drei. Erst an vierter Stelle - und mit großem Abstand - kam die EU-25 mit einem prozentualen Weltmarktanteil von 6,9\% (VSM 2006).

Die Dominanz Südkoreas und Japans in der zweiten Hälfte des 20. Jahrhunderts führte zu einem dramatischen Werftensterben und einem drastischen Rückgang der Zahl der Werftbeschäftigten in Europa. In Deutschland sank sie beispielsweise zwischen 1975 und 1995 von 106.000 auf 33.000. Auch in den darauf folgenden Jahren setzte sich der Beschäftigungsabbau weiter fort, sodass heute nur noch ca. 20.000 Menschen auf den deutschen Werften arbeiten (-31 \% gegenüber 1995; Ludwig/Tholen 2006). Ähnlich verlief die Entwicklung in den anderen europäischen Schiffbaunationen.

Allerdings verursachten nicht nur die Überlegenheit Japans und Südkoreas im Falle Südkoreas gestärkt durch vermutetes Dumping -, sondern auch Produktivitätsfortschritte, das heißt, eine intensive Automatisierung und die Einführung arbeitsorganisatorischer Innovationen, den Beschäftigungseinbruch. Hinzu kam der gestiegene Grad der Arbeitsteilung zwischen Werften als Endproduzenten und der Zulieferindustrie. Werften decken einen immer geringeren Anteil der Wertschöpfungskette selbst ab, was seinen Ausdruck darin findet, dass heutzutage ca. $70 \%$ eines Schiffsneubaus von Zulieferern erbracht werden. Die Werften übernehmen dabei zunehmend die Rolle des "Systemintegrators“, der das Design, die Produktionsabläufe, die Finanzierung und das Funktionieren der Zulieferkette verantwortet.

\subsection{STRUKTUR DER BRANCHE}

Auf den 240 Werften, die in den 20 europäischen Ländern mit dem Neubau von Seeschiffen oder deren Reparatur befasst sind, arbeiteten im September 2004 insgesamt 154.872 Menschen - hiervon ist der Großteil in den Mitgliedstaaten der EU-25 (127.489 Beschäftigte) anzutreffen. Differenziert nach alten und neuen EU-Mitgliedstaaten (ohne Rumänien und Bulgarien) zeigt sich, dass 97.808 Menschen in der alten EU-15 und 29.681 Menschen in den neuen EU-10 Staaten auf Werften Beschäftigung fanden. ${ }^{2}$

Die nähere Betrachtung der Struktur der europäischen Werftbeschäftigung zeigt, dass ein Großteil der Gesamtbeschäftigung auf wenige Werftengruppen entfällt, die über Standorte in mehreren europäischen Ländern verfügen. Auf insgesamt 80 Werften, die zu zehn Werftkonzernen gehören, waren 2004 insgesamt 87.100 Menschen beschäftigt. Das bedeutet, dass auf $34 \%$ aller europäischen Werften insgesamt $56 \%$ der gesamten europäischen Werftbeschäftigten angestellt sind. Die norwegische Aker Yards Gruppe mit ihren 13 Werften in fünf europäischen Ländern ist der mit Abstand größte europäische Werftenkonzern. Er beschäftigt insgesamt ca. 16.000 Schiffbauer. Europäisch aufgestellt sind auch die dänische Odense Shipyard Group (5.000 Beschäftigte an vier Standorten), die niederländische Damen Shipyard Group (9.700 Beschäftigte an 20 Standorten) und die deutsche ThyssenKrupp Marine Systems (10.000 Beschäftigte an 8 Standorten). Darüber hinaus existieren in Europa noch weitere große Werftengruppen, die jedoch im engeren Sinne nicht als ,europäische" Konzerne bezeichnet werden können, da sie zumeist nur über Standorte in ihren jeweiligen Heimatländern verfügen.

\subsection{SPEZIALISIERUNG AUF HIGH-TECH-SCHIFFBAU}

Der seit den 1980er Jahren stagnierende bzw. sinkende Marktanteil Europas an der Weltschiffbauproduktion darf nicht darüber hinwegtäuschen, dass Europa sich in den letzten Jahrzehnten in bestimmten Marktsegmenten eine herausragende Stellung erobern konnte. Vor allem der Bau von High-Tech-Schiffstypen, wie Kreuzfahrtschiffe, Marineschiffe, Roll on/Roll off-Fähren, Spezial- und Arbeitsschiffe, ist dabei nach wie vor eine Domäne der Europäer: Bei Kreuzfahrtschiffen teilen die drei europäischen Anbieter, die Meyerwerft, Fincantieri und Aker Yards, den Weltmarkt unter sich auf; bei den Roll on/Roll off-Fähren sind die Europäer mit Abstand Weltmarktführer und auch auf dem offenen Markt für Marineschiffe (vor allem bei U-Booten, Fregatten, Korvetten) nimmt die europäische Schiffbauindustrie eine Spitzenposition ein. Aber auch technologisch weniger anspruchsvolle Schiffstypen, wie z. B. Containerschiffe, werden weiterhin in Europa gebaut. So produziert beispielsweise die dänische Odense Shipyard die weltweit größten Containerschiffe mit einer Stellkapazität von bis zu 13.000 Standardcontainern.

\section{Kooperationsstrukturen und Qualifikation}

\subsection{WEST-OST-KOOPERATION}

Kooperationen zwischen den Werften in Europa, zwischen Werften und Zulieferern sowie die Zusammenarbeit zwischen Werften und wissenschaftlichen Einrichtungen sind Schlüsselelemente der europäischen Schiffbauindustrie, um den globalen Wettbewerbsanforderungen

\footnotetext{
2 Gemessen am Volumen der Neubauaufträge im Zeitraum 2002 bis 2005 ist Deutschland mit knapp $26 \%$ aller Aufträge die mit Abstand erfolgreichste Schiffbaunation innerhalb Europas. Danach folgen Italien (11\%), Kroatien (11\%), Polen (10\%), Dänemark (8\%), Rumänien (6\%) und Finnland (5\%).
} 
in der Zukunft gerecht werden zu können. ${ }^{3}$

Nachdem durch den Kollaps der sozialistischen Systeme Osteuropas der im Rahmen des Comecon organisierte Schiffbaumarkt zusammengebrochen war, mussten sich die mittel- und osteuropäischen Werften der Konkurrenz auf dem Weltmarkt stellen, was angesichts des technologischen Rückstands der dortigen Werften nur wenig Erfolg versprechend zu sein schien. Auf einen „Investitions- und Modernisierungsschub", wie ihn die ostdeutschen Werften nach 1990 erfahren haben, konnten die Werften in Polen, Rumänien, Kroatien oder im Baltikum nicht hoffen. Jedoch entwickelte sich eine Arbeitsteilung zwischen Ost und West, die dem osteuropäischen Schiffbau Perspektiven zumindest in rudimentärer Form eröffnen konnte und zugleich für die westeuropäischen Werften Vorteile bot.

Die Form der Zusammenarbeit zwischen west- und osteuropäischen Werften besitzt heute weniger den Charakter einer Kooperation zwischen gleichen Partnern, vielmehr muss sie als Sub-Contracting charakterisiert werden. Fast die Hälfte aller befragten Handelsschiffsneubauwerften in Westeuropa gibt an, mit Werften in Mittelund Osteuropa zusammenzuarbeiten. Deutlich geringer fiel der Wert bei den Marineschiffswerften (19\%) und den Reparaturwerften (33\%) aus. Über alle Werfttypen hinweg zeigt sich dabei, dass die mittel-/osteuropäischen Werften vor allem als verlängerte Werkbank westeuropäischer Werften fungieren, denn die Zusammenarbeit findet beinahe ausschließlich im Bereich der Stahlarbeiten statt. Westeuropäische Werften lassen hierbei die Schiffshüllen, Deckaufbauten oder Lukendeckel in Mittel-/Osteuropa fertigen und zur Zusammenstellung des Schiffs auf die westlichen Werften transportieren. In wissensintensiven Arbeitsbereichen wie Konstruktion, Ausrüstung, Design, F\&E oder Marketing findet eine Kooperation - wenn überhaupt - nur auf einem sehr geringen Niveau statt. Die Konzentration auf den Bereich Hüllenbau/Stahlbau zeigt, dass hier die westeuropäischen Werften Kostenvorteile zu nutzen suchen, die in den geringeren Arbeitskosten in Mittel-/Osteuropa begründet sind. Diese Mischkalkulation macht die Produkte insgesamt wettbewerbsfähiger.

Im Bereich des Handelsschiffbaus ist jedoch auch der Typus der ausländischen Di- rektinvestition anzutreffen. Dies ist vornehmlich der Fall bei der Kooperation der Aker Werften mit Rumänien, der Damen Werften mit Rumänien und Polen und der A. P. Moeller-Maersk-Werften mit Estland und Litauen. Dort findet die Kooperation quasi konzernintern statt, da die Partnerwerften in Mittel-/Osteuropa sich im Eigentum der westeuropäischen Mutterkonzerne befinden - doch auch in diesen Fällen ist die Nutzung von Lohnkostenvorteilen in Mittel- und Osteuropa das dominante Motiv. Nach den Ergebnissen unserer Befragung beliefen sich die durchschnittlichen jährlichen Kosten für einen ausgebildeten Schiffbauer im Jahr 2004 in Deutschland auf $48.800 €$, in den Niederlanden auf $40.600 €$, in Dänemark auf $40.500 €$, in Kroatien auf $14.500 €$, in Polen auf $9.700 €$ und in Rumänien auf nur $3.900 €{ }^{4}$

\subsection{KOOPERATION INNERHALB WESTEUROPAS}

Hingegen sind die Kooperationsbeziehungen der Werften innerhalb Westeuropas anders zu bewerten. Es handelt sich - wie die Kooperationsinitiativen Intership und Euroyards belegen - um eine intensive Form der Kooperation unter gleichberechtigten Partnern, die vor allem in wissensintensiven und sensiblen Bereichen stattfindet. Diese zwei Initiativen wurden ins Leben gerufen, um die Einkaufsaktivitäten sowie Forschungs- und Entwicklungsaktivitäten der beteiligten Werften zu bündeln und damit Kostenvorteile zu erzielen. Beispielsweise sind an Euroyards Fincantieri (Italien), die seit 2006 zur Aker-Gruppe gehörende Chantiers de l'Atlantique (Frankreich), die zum TKMS-Konzern gehörende HDW GmbH und die Jos. L. Meyer Werft (beide Deutschland), IZAR (Spanien) und Aker Finnyards (Finnland) beteiligt. Hier hat sich die Kooperation um das Segment der Kreuzfahrtschiffe herum entwickelt, denn die beteiligten Werften stehen bei der Entwicklung und dem Bau von Passagierschiffen vor identischen Herausforderungen. Die Einrichtung von Euroyards kann als Beleg dafür gelten, dass Kooperation auch dann funktionieren kann, wenn die Partner auf demselben Markt in Konkurrenz zueinander stehen.

Dass die interne westeuropäische Werftenkooperation einen vollständig anderen Charakter hat als die Kooperation mit Mittel-/Osteuropa, zeigt sich auch in den Bereichen der Zusammenarbeit. Hier findet vor allem auf den wissensintensiven Gebieten ein enger Austausch statt. Beim Design ist die Kooperation zwischen den westeuropäischen Werften am intensivsten. Ebenfalls bemerkenswert ist der Befund, dass in den Bereichen F\&E, Marketing und Konstruktion die Kooperation auf einem hohen Niveau stattfindet. Beim Vergleich der einzelnen Werfttypen fällt auf, dass im Marineschiffbau die Kooperation über alle Bereiche hinweg am stärksten ausgeprägt ist. Die verschiedenen grenzüberschreitenden Entwicklungs- und Produktionsprojekte beispielsweise zwischen Navantia und DCN, BAE Systems und DCN, Fincantieri und Navantia, ThyssenKrupp Marine Systems und Navantia deuten auf eine intensive Vernetzung in diesem Segment hin.

\subsection{ZWISCHENFAZIT}

Eine strategische Kooperation findet somit lediglich innerhalb Westeuropas statt und geht weit über das reine Outsourcing von einfachen Arbeiten hinaus, welches das zentrale Motiv bei der Kooperation Westeuropas mit Mittel-/Osteuropa ist. In diesen Fällen hat die Zusammenarbeit eher den Charakter einer Auftragsvergabe an einen Zulieferer.

Die Zusammenarbeit innerhalb Westeuropas konzentriert sich hauptsächlich auf wissensintensive Bereiche, was als Zeugnis für die hohe Standardisierung und die einheitlichen Qualitätsstandards im westeuropäischen Schiffbau betrachtet werden kann. Bei der Zusammenarbeit westeuropäischer mit den mittel-/osteuropäischen Werften treten dagegen häufig Probleme technischer Natur auf, die Auswirkungen auf Ablieferungstermine oder Qualitätsansprüche haben. Hier scheint es seitens der mittel-/osteuropäischen Partnerwerften noch einen zum Teil erhebli-

3 Im Folgenden konzentrieren wir uns auf die horizontale Kooperation, bei der wir den größten Handlungsbedarf vermuten. Demgegenüber ergab sich aus unserer Erhebung ein hoher Grad der Zusammenarbeit zwischen den Werften und den Zulieferern in allen Bereichen.

$4 \quad$ Bei einem Vergleich der einzelnen Länder muss auch der Faktor der Produktivität berücksichtigt werden. Dies konnte im Rahmen der Untersuchung jedoch nicht abgedeckt werden. Für den Vergleich zwischen Polen und Deutschland kamen unveröffentlichte Kalkulationen jedoch zu dem Ergebnis, dass die Produktivität der polnischen Werften ca. $50 \%$ unter der der deutschen Werften liegt. 
chen Nachholbedarf zu geben, um die Qualitätsstandards des westeuropäischen Schiffbaus zu erreichen.

Andererseits werden jedoch trotz aller Fortschritte in der Kooperation die Chancen einer erweiterten Zusammenarbeit noch nicht hinreichend genutzt. Zwar existieren institutionalisierte Instrumente für eine horizontale Zusammenarbeit. Gleichwohl werden sie nur von einer vergleichsweise geringen Zahl von Akteuren aktiv genutzt. Die Kostenvorteile, die z. B. durch einen gemeinsamen Einkauf standardisierter Bauteile oder der koordinierten Entwicklung neuer Technologien generiert werden könnten, werden nur unzureichend ausgeschöpft. Es dominieren immer noch kurzfristige Strategien, die den Aufbau langfristiger Kooperationsstrukturen behindern.

\section{Personalkrise}

\section{1 ...IN DEUTSCHLAND}

In nahezu allen Ländern besteht ein Mangel an qualifizierten Fachkräften. Auffällig ist dabei, dass diese Probleme nicht nur im Bereich der Ingenieure bzw. Hochschulabsolventen auftreten, sondern fast alle Gewerke der Werften betreffen.

Die Krisen und Produktionsschwankungen des europäischen Schiffbaus sowie das Image einer 3-D-Industrie (3D: Dirty, Difficult and Dangerous) haben dieser Branche den Stempel einer niedergehenden und rückständigen Industrie aufgedrückt. Dass es sich tatsächlich um eine High-TechIndustrie handelt, ist in der Öffentlichkeit nur wenig bekannt. Dies hat unter anderem dazu geführt, dass sich junge Menschen nicht mehr für den Schiffbau interessieren. Bei Werften, die auf dem (regionalen) Arbeitsmarkt in direkter Konkurrenz zu anderen High-Tech-Branchen wie der Luftfahrt- oder Automobilbranche stehen, kommt noch hinzu, dass insbesondere Ingenieure dort ein deutlich höheres Einkommen erzielen können als auf den Werften.

Neben dem schlechten Image gibt es aber auch noch „hausgemachte" Ursachen für die gegenwärtige Personalkrise, nämlich das von den meisten Werften verfolgte Konzept der „Stammbelegschaft": Eine zu geringe Stammbelegschaft führte zu einem intensiven Einsatz von Leiharbeit und zu einer Externalisierung der Ausbildung qualifizierter Fachkräfte. So finden sich auf deutschen Werften häufig Leiharbeitsquoten von über $50 \%$ (Ludwig/Tholen 2006, S. 29f.). Bis vor wenigen Jahren konnten sich die Werften noch darauf verlassen, von den Entleihfirmen qualifiziertes Personal zu erhalten, da dort viele ehemalige Schiffbauer nach dem Beschäftigungsabbau auf den Werften untergekommen waren. Mittlerweile müssen die Werften jedoch zunehmend nachschulen und intensiver einweisen, was zum Teil erhebliche Zusatzkosten, Störungen im Arbeitsablauf und Frustrationen bei der Stammbelegschaft hervorruft.

Dem Schiffbau droht in Zukunft demnach anstatt einer Auslastungskrise eine Personalkrise, welche die Innovationsfähigkeit der Werften gefährdet. So geht der Verband für Schiffbau und Meerestechnik e.V. (VSM) davon aus, dass auf den deutschen Werften der jährliche Bedarf an Ingenieuren zwischen 120 und 125 Personen liegt (Die Welt vom 5.8.2006). In den vergangenen 20 Jahren sei der Anteil von Ingenieuren auf den Werften von 3 auf mehr als $12 \%$ gestiegen. Ob dieser Bedarf mittelfristig befriedigt werden kann, erscheint mehr als fraglich. Zwar sind im Jahr 2006 ca. 900 Studenten an sechs Hochschulen in der Fachrichtung Schiffbau-Ingenieur eingeschrieben. Doch die Zahl der Absolventen dürfte deutlich darunter liegen, denn allein an der TU-Harburg geht man davon aus, dass nur $40 \%$ dieses Studium auch tatsächlich beenden (ebd.). Nachwuchsprobleme gibt es jedoch auch im Bereich der Facharbeiter. Angesichts der schiffbautypischen Konjunkturschwankungen müssten die Werften ihren Nachwuchs antizyklisch ausbilden. In der Realität findet dies jedoch nicht statt. Zwar haben z. B. die deutschen Werften auf der Nationalen Maritimen Konferenz im Dezember 2006 angekündigt, die Zahl der Auszubildenden um $10 \%$ zu erhöhen. Diese Auszubildenden stehen den Werften allerdings erst in vier bis fünf Jahren als qualifizierte Fachkräfte zur Verfügung. Angesichts der akuten Personalprobleme des deutschen wie europäischen Schiffbaus scheint diese Initiative zu spät zu kommen, gleichwohl ist sie unerlässlich.

\section{$4.2 \ldots$ IN ANDEREN LÄNDERN}

Der kaum noch zu deckende Bedarf an qualifizierten Mitarbeitern trifft nicht nur die deutschen Werften. Die Werften in Nor- wegen, Finnland, Großbritannien, aber auch in Polen und Rumänien haben mit ähnlichen Schwierigkeiten zu kämpfen. Vor allem in Großbritannien geht man davon aus, dass angesichts der bald aus Altersgründen ausscheidenden Werftarbeiter in den nächsten Jahren ein Personalbedarf im Schiffbau von über 5.000 Personen entstehen wird (BBC News vom 30.7.2006; Birkler et al. 2002). Durch die gegenwärtigen Ausbildungsprogramme wird dieser Bedarf kaum gedeckt werden können, und auch die ehemals auf den Werften beschäftigten Mitarbeiter, die im Verlauf der Werftenkrise in den 1980er und 1990er Jahren entlassen worden sind, werden nicht mehr für den Schiffbau zu mobilisieren sein. Studien haben im Falle der schottischen Werften gezeigt, dass $70 \%$ derjenigen, die einmal den Schiffbau verlassen haben, nicht mehr in die Branche zurückkehren. ${ }^{5}$

Während man in Großbritannien noch darüber nachdenkt, in großem Maße den Personalbedarf durch ausländische Arbeitskräfte zu decken (Lloyd's List vom 17.7.2006), ist man in Norwegen oder Finnland schon weiter. Dort ist man angesichts des fehlenden einheimischen Nachwuchses bereits dazu übergegangen, Werftarbeiter aus osteuropäischen Staaten wie Polen oder dem Baltikum anzuheuern selbstverständlich zu deutlich geringeren Löhnen. Als Konsequenz haben bereits heute polnische Werften Probleme, die Abwanderung polnischer Fachkräfte in den Westen zu kompensieren. Wie in Norwegen, Finnland oder Großbritannien richtet man nun auch in Polen den Blick gen Osten und wirbt verstärkt ukrainische Werftarbeiter an - nachdem im Sommer 2006 bereits nordkoreanische Arbeiter auf den Werften angeheuert hatten (BBC News vom 13.1.2006). In Rumänien fördert man angesichts der Rekrutierungsschwierigkeiten und der starken Abwanderung gen Westen verstärkt die Einstellung von Frauen in klassischen Schiffbaugewerken, da man von ihnen nicht erwartet, dass sie so schnell das Land verlassen wie die Männer. In einigen baltischen Ländern mussten die Werften im vergangenen Jahr bis zu

Die britische Regierung hat darauf reagiert und zusammen mit den Arbeitgebern Ende 2006 die National Skills Academy Network gegründet, um dem akuten Fachkräftemangel in den Bereichen Manufacturing, Construction und Financial Services zu begegnen. 
$60 \%$ ihres Personals aufgrund von Abwanderung zu westeuropäischen Unternehmen ersetzen. Dies führte zu Lohnerhöhungen von bis zu $50 \%$, um den verbliebenen Arbeitern einen Anreiz zum Bleiben zu geben.

Die Gesamtentwicklung zeigt somit in aller Deutlichkeit, dass sich die europäische Schiffbauindustrie als Ganzes verstärkt der Personalentwicklung widmen muss. Die Entwicklung führt mittelfristig zu einer Aushöhlung der Fachkräftebasis in Mittelund Osteuropa und verursacht zudem hohe Integrationskosten auf den westeuropäischen Werften. Ohne ein abgestimmtes Konzept auf diesem Gebiet bleibt die Zukunftsfähigkeit des Schiffbaustandortes Europa grundlegend gefährdet.

Angesichts der zweifelhaften Preisgestaltung südkoreanischer und chinesischer Konkurrenten können europäische Werften nur überleben, wenn sie das bessere Schiff anbieten. Für die Zukunft dieser High-Tech-Branche ist deshalb eine proaktive Personal- und Nachwuchspolitik unverzichtbar.

\section{5 \\ Europäische Antworten}

\subsection{INITIATIVEN DER INDUSTRIE}

Wegen der Defizite des europäischen Schiffbaus und des zunehmenden Wettbewerbsdrucks durch den Ausbau von Schiffbaukapazitäten in Fernost sind in erster Linie die Werften selbst aufgefordert, die Strukturen des europäischen Schiffbaus durch eine intensivere Kooperation zu verbessern.

Die Initiativen Intership und Euroyards sind erste gute Ansätze, doch sie reichen bei weitem nicht aus. Erforderlich ist der Aufbau einer belastbaren Vertrauenskultur. Dies benötigt jedoch Zeit, die angesichts der gegenwärtigen komfortablen Auftragslage im Prinzip auch zur Verfügung steht.

Auf europäischer Ebene hat die Industrie mit Unterstützung der Gewerkschaften die industriepolitische Initiative LeaderShip 2015 (CESA 2003) angestoßen, die erstmals die bis dahin nationalstaatlichen Schiffbaupolitiken zu überwinden sucht und den Schiffbau - auch angesichts seiner strategischen Bedeutung für Europa - als eine gesamteuropäische Aufgabe begreift. Die Initiative zielt auf die Schaffung fairer
Wettbewerbsbedingungen und setzt in diesem Zusammenhang auf die Einhaltung der Regularien der Welthandelsorganisation (WTO) und den Abschluss eines OECD-Abkommens für den Schiffbau. Sie verfolgt aber auch eine Intensivierung der Forschungs- und Entwicklungsaktivitäten, um den immer noch bestehenden Technologievorsprung gegenüber den ostasiatischen Konkurrenten aufrechterhalten bzw. ausbauen zu können. Ein fester Bestandteil dieser Initiative sind zudem gemeinsame Anstrengungen aller europäischen Schiffbaunationen im Bereich der Personalentwicklung und der Qualifikation. LeaderShip 2015 soll dabei nicht nur auf EU-Ebene handlungsleitend sein, sondern auch auf den jeweiligen nationalen Ebenen vermarktet werden. Mit der Umsetzung von LeaderShip 2015 auf den nationalen Ebenen ist erst begonnen worden, und es bleibt abzuwarten, ob angesichts der dramatischen Entwicklung im Weltschiffbau die verbleibende Zeit ausreicht, nachhaltige Kooperationsstrukturen in den einzelnen Ländern zu entwickeln.

\subsection{DIE ROLLE DES SOZIALEN DIALOGS}

Die EU-Politik besaß bislang eher einen flankierenden Charakter, der bestehende Entwicklungen begleitete. Die strukturellen Defizite im Bereich der Kooperation und der Qualifikation im europäischen Schiffbau erfordern auf der EU-Seite jedoch ergänzend dazu aktivierende Elemente, welche bislang kaum erkennbar sind.

Vielversprechend erscheint der im Jahr 2003 ins Leben gerufene sektorale Soziale Dialog Schiffbau, der vonseiten der EUKommission unterstützt wird. Die gemeinsame Arbeit der Arbeitgeberverbände und der Gewerkschaften in diesem Gremium ermöglicht vor allem die Generierung eines gemeinsamen Verständnisses des europäischen Schiffbaus. Angesichts der nach wie vor nationalstaatlich geprägten Politiken in den EU-Mitgliedstaaten, muss der Soziale Dialog als eine Grundvoraussetzung zur Überwindung dieser „Nationalismen“ betrachtet werden. Insbesondere zur Verbesserung des Images und zur Bewältigung der Personal- und Qualifikationsprobleme wurden bereits erste Anstrengungen unternommen. So haben sich die Sozialpartner nicht nur bemüht, ein gemeinsames Verständnis für die Ursachen der alle europäischen Schiffbaunationen betreffenden Per- sonalprobleme zu gewinnen. Es wird auch nach Wegen gesucht, wie beispielsweise die Ausbildung im Schiffbau europaweit standardisiert werden könnte. Darüber hinaus sollen best practice Fälle von innovativer und flexibler Personalpolitik ausgewählt werden, an denen sich die Werften in Europa orientieren können. Um aus den im Sozialen Dialog gewonnenen Erkenntnissen jedoch Realpolitik werden zu lassen, müsste dieses Gremium in die Lage versetzt werden, verbindliche Regelungen für die Industrie zu vereinbaren. Diese hätten bei allen Akteuren eine hohe Akzeptanz, da sie sozialpartnerschaftlich entwickelt worden wären.

\subsection{ELEMENTE EINER MARITIMEN INDUSTRIEPOLITIK}

Erste Ansätze einer maritimen Industriepolitik der EU (zum Beispiel durch das Grünbuch vom Juni 2006) existieren bereits. Diese Politikansätze tragen durchaus Züge einer modernen Industriepolitik im Sinne einer dialogorientierten Netzwerkpolitik (Seidel 1999, S. 135). Sie sind aber nicht primär von der Industrie (also im Falle des Schiffbaus von den Werften und den hier vertretenen Sozialpartnern), sondern eben „nur“ von der Kommission bestimmt.

Infolgedessen ist die Kommission gezwungen, die unterschiedlichen Standortbedingungen, Strukturen und auch Interessen der Werften von „oben“ auszugleichen, statt dass - gemäß dem EU-Politikverständnis der Subsidiarität - zunächst vor allem die Werften selbst ihrer Verpflichtung zu notwendigen Veränderungen auf Betriebsebene und zur Kooperation zwischen den einzelnen Werften nachkommen würden. Erst dann, wenn also die Werften in Europa ihre „Hausaufgaben“ gemacht hätten, könnte eine maritime Industriepolitik der EU wirksamer durchgesetzt werden und träfe auch auf eine höhere Akzeptanz in den jeweiligen Mitgliedsländern.

Die Differenziertheit der europäischen Werftenlandschaft und die unterschiedliche Qualität der Zusammenarbeit in den einzelnen Bereichen und auf den unterschiedlichen Ebenen erfordern eine strategische Netzwerkbildung. Hierzu müsste die EU Ansätze entwickeln, um eine Beteiligung der Akteure an diesem Prozess der Netzwerkbildung zu erreichen. Eine aktivierende Schiffbaupolitik könnte unter an- 
derem den Zugriff auf existierende Fördermechanismen und spezifische Forschungsprogramme an Bedingungen knüpfen. Die Teilnahme an Kooperationsinitiativen oder nachgewiesene Anstrengungen im Bereich der vorausschauenden Personalpolitik könnten als Eingangsvoraussetzung definiert werden, um in den Genuss von EUFördermitteln zu gelangen.

Die bislang eher zurückhaltende Rolle der maritimen Industriepolitik auf europäischer Ebene ist jedoch politisch begründet. Sie trägt den Bedenken der europäischen Wettbewerbspolitik und den Grundregeln der WTO Rechnung. Vor allem aber ist sie den nationalstaatlichen Egoismen der EU-Schiffbauländer geschuldet.

Konzepte wie die der „systemischen Wettbewerbsfähigkeit“ und des „dialog- orientierten Netzwerkes" mit ihren zentralen Funktionsmechanismen, wie Selbstorganisation, gemeinsames Ziel, soziale Funktionslogik, Verbindung mit hoheitlichen Systemen, komplexe Umgebung und Strategiefähigkeit (Seidel 1999, S. 8; Messner 1995), müssen zunächst von den Werften selbst - und nicht vom Staat entwickelt und auch angewendet werden. Sie sind eine notwendige Voraussetzung für eine maritime Industriepolitik der EU-Kommission. Daran anschließend kann eine Politik der EU „greifen“, die vom Prinzip der „offenen Koordinierung“ geleitet wird. Dieses Prinzip würde durchaus die Bildung und/oder industriepolitische Förderung von Netzwerken im europäischen Schiffbau ermöglichen, welche dann nicht alle Werftstandorte gleichermaßen umfassen müssten. Und sie würde auch den Erfordernissen der Wettbewerbspolitik Rechnung tragen.

Als Fazit bleibt jedoch festzuhalten: Solange die privaten Akteure (also die Schiffbauunternehmen) ihre gemeinsamen Probleme innerhalb der EU nicht energisch genug selbst erfolgreich bearbeiten können oder wollen, bleibt jede EU-Industriepolitik unvollständig, hochgradig fragil und zeitlich sehr aufwendig. Gerade in einer Zeit, in der die Werften in Europa (wenn auch zum Teil sehr unterschiedlich) über eine gute Auslastung verfügen, wäre ihnen die Möglichkeit gegeben, ihre „Hausarbeiten" zu machen, ehe ihnen - voraussichtlich in der zweiten Dekade unseres Jahrhunderts - wieder der schärfere Wind des globalen (ostasiatischen) Wettbewerbs die Luft zu tief greifenden Strukturveränderungen nehmen könnte.

\section{LITERATUR}

BBC News (2006): Gdansk appeal for Ukraine workers; 13.1., www.newsvote.bbc.co.uk/mapps/pagetools/print/news/bbc.co.uk BBC News (2006): Shipyards in appeal for skilled workers; 30.7. www.eveningtimes.co.uk/print/news/5054982.shtm Birkler, J./Schlank, J. F./Arena, M./Smith, G. K./ Lee, G. (2002): The Royal Navy's New-Generation Type 45 Destroyer: Acquisition Options and Implications; Rand Corporation, London

CESA (2003): LeaderShip 2015, Brüssel CESA (2006): Annual Report 2004-2005, Brüssel

Damen, K. (2003): The Damen Shipyard Group - Presentation at the Dutch Shipbuilding Conference, Driebergen

Die Welt (2006): Keine Chance für Rumhänger, 5.8.

EMF - European Metalworker's Federation (Hrsg.) (2006): Industriewachstum in China: Europäische Industrien im Aufruhr, Brüssel Kommission der Europäischen Gemeinschaften (2006): GRÜNBUCH Die künftige Meerespolitik der EU: Eine europäische Vision für Ozeane und Meere, Brüssel

Lawton, Th. C. (Hrsg.) (1999): European industrial policy and competitiveness: concepts and instruments, St. Martins Press, New York
Lloyd's List (2006): Skills crisis may force UK yards to look abroad for expertise; 17.7., www.lloydslist.com

Messner, D. (1995): Die Netzwerkgesellschaft. Wirtschaftliche Entwicklung und internationale Wettbewerbsfähigkeit als Problem gesellschaftlicher Steuerung, Köln

Ludwig, Th./Tholen, J. (2006): Beschäftigung, Auftragslage und Perspektiven im deutschen Schiffbau - Ergebnisse der 15. Betriebsrätebefragung im September 2006, IAW-Forschungsbericht 12, Bremen

Porter, M. E. (1991): Nationale Wettbewerbsvorteile. Erfolgreich konkurrieren auf dem Weltmarkt, München

Seidel, K. (1999): Maritime Netzwerke und europäische Industriepolitik, Dissertation Universität Bremen, Frankfurt/M.

Tholen, J./Ludwig, Th. (2006): Projektbericht - Schiffbau in Europa, November 2006, Bremen (im Erscheinen)

VSM - Verband für Schiffbau und Meerestechnik (2006): Jahresbericht 2005, Hamburg, www.welt.de/data/2006/08/05/985826.html 